\title{
Candida lusitaniae as an unusual cause of recurrent vaginitis and its successful treatment with intravaginal boric acid
}

\author{
Neil S. Silverman', Margie Morgan² and W.S. Nichols² \\ ${ }^{1}$ Department of Obstetrics and Gynecology, Cedars-Sinai Medical Center, \\ Burns \& Allen Research Institute, UCLA School of Medicine, Los Angeles, CA \\ ${ }^{2}$ Department of Pathology and Laboratory Medicine, Cedars-Sinai Medical Center, \\ Burns \& Allen Research Institute, UCLA School of Medicine, Los Angeles, CA
}

Increasing use of short-course antifungal therapies in patients with recurrent vulvovaginitis may enable the emergence of less-common, more resistant yeast strains as vaginal pathogens. We report the case of a patient with chronically symptomatic and repeatedly treated vaginal candidiasis whose infection was attributable to Candida lusitaniae, a previously unreported cause of candidal vaginitis.

Key words: Vulvovaginitis; VAginitis; CANDidA; Boric ACID

\section{CASE REPORT}

A 55-year-old gravida 4, para 2022 Caucasian woman was referred for evaluation and management of chronic recurrent vulvovaginitis. The patient reported approximately five years of vaginal symptoms consisting primarily of 'burning' and 'itching', with 'outbreaks' occurring every 1-3 months. She had been treated, in response to symptoms and culture results, with a variety of systemic and topical antimicrobials and antifungals over the past years. Her most recent antifungal therapy was 6 months prior to referral, and consisted of a two-dose course of oral fluconazole with moderate symptom relief. Approximately 1 month prior to referral, the patient had been treated with a seven-day course of intravaginal clindamycin cream after a vaginal culture was positive for Gardnerella vaginalis; she reported only 1-2 days of symptomatic relief after that therapy.
The patient was in good health, with no chronic medical conditions. She was taking no medications. A total abdominal hysterectomy with ovarian preservation had been performed 17 years earlier for uterine leiomyomata. The patient reported no menopausal vasomotor symptoms, and was not taking hormone replacement therapy. Evaluation of follicle-stimulating hormone (FSH) and luteinizing hormone (LH) levels had recently been performed in her referring doctor's office and were not in the menopausal range.

At the time of initial evaluation, the patient reported only minimal symptomatology. Examination revealed no redness or swelling of the external genitalia or the vagina. No lesions or excoriations were present. The vaginal $\mathrm{pH}$ was 4.5 , and microscopic examination of the vaginal secretions showed only normal-appearing epithelial cells with scattered large rod-form bacteria. No

Correspondence to: Neil S. Silverman, Department of Obstetrics and Gynecology, Cedars-Sinai Medical Center, 8700 Beverly Boulevard, Suite 160W, Los Angeles, CA 90048. Email: silvermann@cshs.org 
treatment was instituted empirically, and vaginal cultures obtained at that visit subsequently returned negative both for yeast and pathogenic bacteria. The patient was instructed to return on symptom recurrence.

The patient returned for symptom recurrence two months later. Examination was unremarkable, vaginal $\mathrm{pH}$ was 4.5 , and wet prep again showed normal-appearing epithelial cells, without evidence of yeast or other pathogens. Vaginal cultures were obtained with the fungal culture positive for yeast, with speciation pending. Bacterial cultures were negative. The patient was started on oral fluconazole to begin at $200 \mathrm{mg}$ every 4 days for three doses as 'induction' therapy, with a plan to begin chronic suppressive therapy if eradication of symptoms and colonization were proven on follow-up ${ }^{1}$. The patient returned after completing her initial treatment, reporting that her symptoms had improved for two days after the first dose, but had then returned and were still present. Repeat examination showed the presence of budding yeast on saline prep, and a course of oral ketoconazole, $100 \mathrm{mg} /$ day, was started. Five days after starting the ketoconazole, the speciation results from the first positive yeast culture returned as Candida lusitaniae, with the more recent cultures again positive for yeast (these subsequently were also identified as C. lusitaniae). The yeast were identified in our laboratory through morphology on cornmeal agar (Hardy Diagnostics, Santa Maria, CA) and inoculation of an API $20 \mathrm{C}$ assimilation strip (bioMerieux, Hazelwood, MO). To eliminate confusion with other yeast species, a maltose fermentation test was performed in the laboratory too, with absence of maltose fermentation confirming the presence of $C$. lusitaniae. The patient was contacted and reported that she was still symptomatic, with no relief from the ketoconazole. Her treatment was changed to boric acid vaginal suppositories, $600 \mathrm{mg}$ nightly for 14 days. The patient's symptoms resolved, and a follow-up vaginal fungal culture two weeks after completing therapy was negative. She developed a reddened, irritated site at the posterior forchette toward the end of therapy, which responded to a topical emollient cream.

\section{DISCUSSION}

While Candida albicans remains the most common yeast species implicated in symptomatic vulvovaginitis, recent reports have described a relative decrease in its proportional impact compared with a relative rise in disease attributable to non-albicans species. One recent review calculated an increase in non-albicans vaginal infections from $10 \%$ in the 1970 s to $21 \%$ through the 1980 s, with Torulopsis glabrata and C. tropicalis demonstrating the largest percentage increases for individual species ${ }^{2,3}$.

The change in breakdown of pathogenic yeast species has significant implications for the treatment of candidal vulvovaginitis. The widening diversity in identified yeast strains is thought to result at least in part from expanding use of short-course topical imidazole antifungals, allowing for the emergence of resistant organisms. C. tropicalis and T. glabrata, for example, have been shown to be resistant to a variety of topical preparations both in vitro and in vivo ${ }^{4,5}$, and C. tropicalis has cell-wall characteristics that make it intrinsically less susceptible to imidazole compounds ${ }^{5}$. Shorter courses of less-effective regimens may also allow for overgrowth of more resistant intravaginal yeast species, as has been shown to be the case with the use of low-dose antifungal prophylaxis against systemic yeast infections in neutropenic or immunosuppressed patients ${ }^{6}$. While the shorter-course therapies for vulvovaginitis may enhance compliance for some patients, they may be insufficient or even detrimental for those women with recurrent symptoms who are then treated with multiple sequential courses of both topical and systemic antifungals, allowing the emergence of lesssensitive non-albicans species.

The present case describes the identification of C. Iusitaniae as the pathogen responsible for the most recent episodes of recurrent vulvovaginitis in a chronically-treated woman, and underscores the increased need to consider such uncommon fungal strains in refractory cases. C. lusitaniae was first identified as an opportunistic human pathogen in 1979 in a patient with acute leukemia ${ }^{7}$, and has been identified as a source of fungemia in only 42 cases reported through $2000^{8,9}$. Two-thirds of 
these cases occurred in immunocompromised patients, and no cases of vaginitis attributable to C. Iusitaniae in otherwise healthy women have been reported to date. Of note is the fact that C. lusitaniae has been associated with breakthrough fungemia in patients being treated with singleagent antifungal regimens, though recent investigators have suggested that, in their experience, the use of fluconazole might be an effective singleagent approach against this pathogen in immunocompetent fungemic patients ${ }^{8}$.

Both fluconazole and ketoconazole proved clinically and microbiologically ineffective against the $C$. Iusitaniae vaginitis seen in our patient. Both agents have been shown to be effective in a high proportion of women with recurrent vulvovaginal candidiasis, though non-albicans species, primarily $T$. glabrata, have been reported to be more difficult to control even with longer-duration regimens of these systemic agents ${ }^{1,10}$. The use of boric acid to treat vulvovaginal candidiasis was first reported in $1974^{11}$, and it has been shown to be effective against refractory non-albicans yeast species, specifically $T$. glabrata ${ }^{12}$. Presented in this case with a non-albicans species associated with a similarly high degree of imidazole resistance, a treatment regimen of intravaginal boric acid capsules as described in Sobel's series was employed, with both symptomatic and bacteriologic cure against C. lusitaniae achieved.

In reporting what we believe to be the first reported case of recurrent vaginitis attributable to C. Iusitaniae, along with its successful treatment with non-azole therapy, we add our concerns to others' over the rise of less-common, more difficult-to-treat yeast species in patients with chronically treated infections. While our patient ultimately responded to intravaginal boric acid therapy, this case demonstrates the value of yeast speciation in difficult recurrent cases, and the ongoing need to investigate newer antifungal therapies in the face of an increasingly diverse spectrum of pathogenic agents, even in immunocompetent individuals.

\section{REFERENCES}

1. Sobel JD. Vulvovaginits. When Candida becomes a problem. Derm Clin NA 1998;16:763-8

2. Odds FC, Webster CE, Riley VC, et al. Epidemiology of vaginal Candida infection: significance of numbers of vaginal yeasts and their biotypes. Eur J Obstet Gynecol Reprod Biol 1987; 25:53-66

3. Horowitz BJ, Giaquinta D, Ito S. Evolving pathogens in vulvovaginal candidiasis: implications for patient care. J Clin Pharmacol 1992;32:248-55

4. Kerridge D, Nicholas RO. Drug resistance in the opportunistic pathogens Candida albicans and Candida glabrata. J Antimicrob Chemother 1986;18 (suppl B):39-49

5. Horowitz BJ. Candidiasis: speciation and therapy. Curr Prob Obstet Gynecol Fertil 1990;8:241-5

6. Meunier-Carpentier F, Kiehn JR, Armstrong D. Fungemia in the immunocompromised host: changing patterns, antigenemia, high mortality. Am J Med 1981;71:363-70
7. Pappagianis D, Collins MS, Hector R, et al. Development of resistance to amphotericin B in Candida lusitaniae infecting a human. Antimicrob Agents Chemother 1979;16:123-6

8. Minari A, Hachen R, Raad I. Candida lusitaniae: a cause of breakthrough fungemia in cancer patients. Clin Infect Dis 2001;32:186-90

9. Blinkhorn RJ, Adelstein D, Spagnuolo PJ. Emergence of a new opportunistic pathogen, Candida lusitaniae. J Clin Microbiol 1989;27:236-40

10. Sobel JD. Recurrent vulvovaginal candidiasis: a prospective study of the efficacy of maintenance ketoconazole therapy. N Engl J Med 1986;315: 1455-8

11. Swate TE, Weed JC. Boric acid treatment of vulvovaginal candidiasis. Obstet Gynecol 1974;43: 893-5

12. Sobel JD, Chaim W. Treatment of Torulopsis glabrata vaginitis: retrospective review of boric acid therapy. Clin Infect Dis 1997;24:649-52 


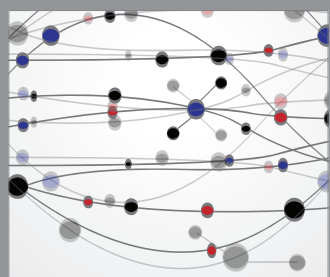

The Scientific World Journal
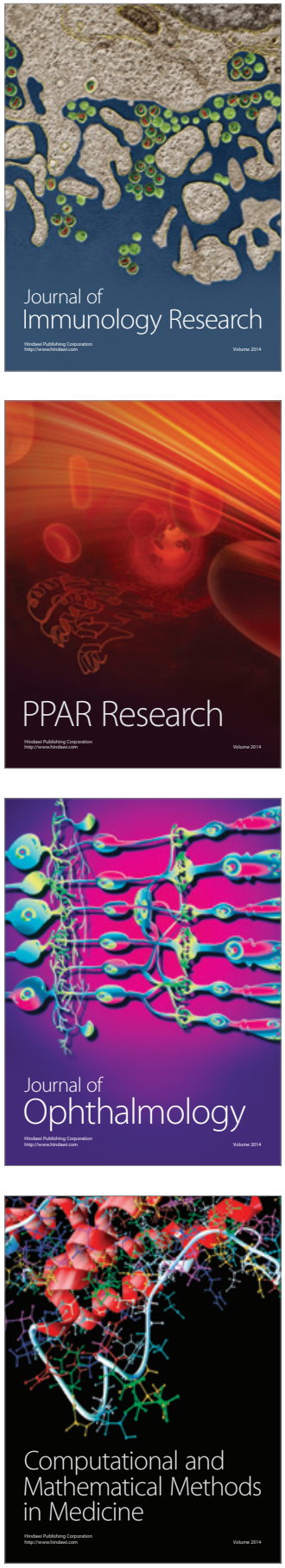

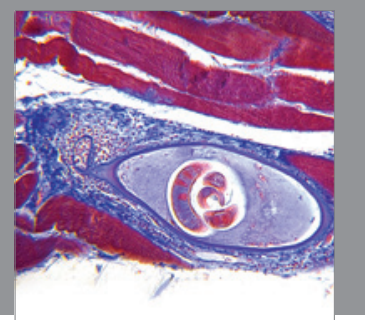

Gastroenterology

Research and Practice
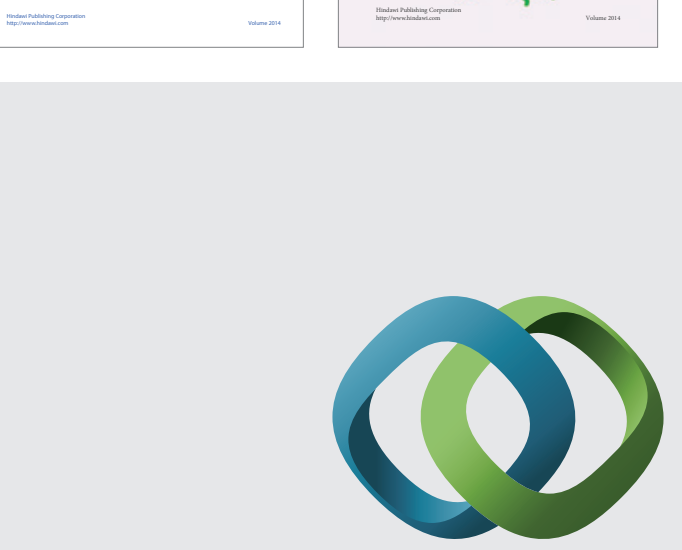

\section{Hindawi}

Submit your manuscripts at

http://www.hindawi.com
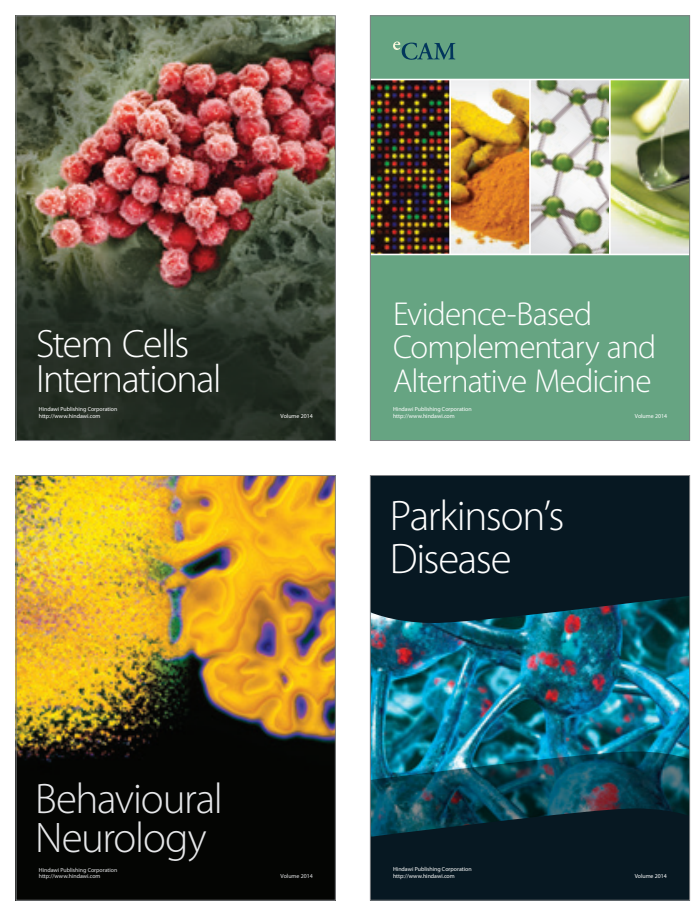

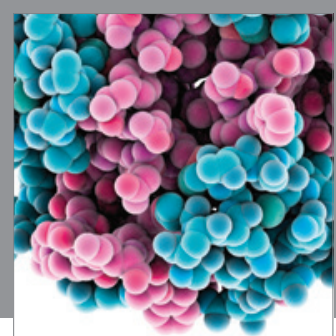

Journal of
Diabetes Research

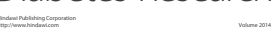

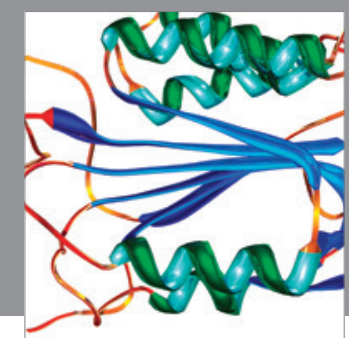

Disease Markers
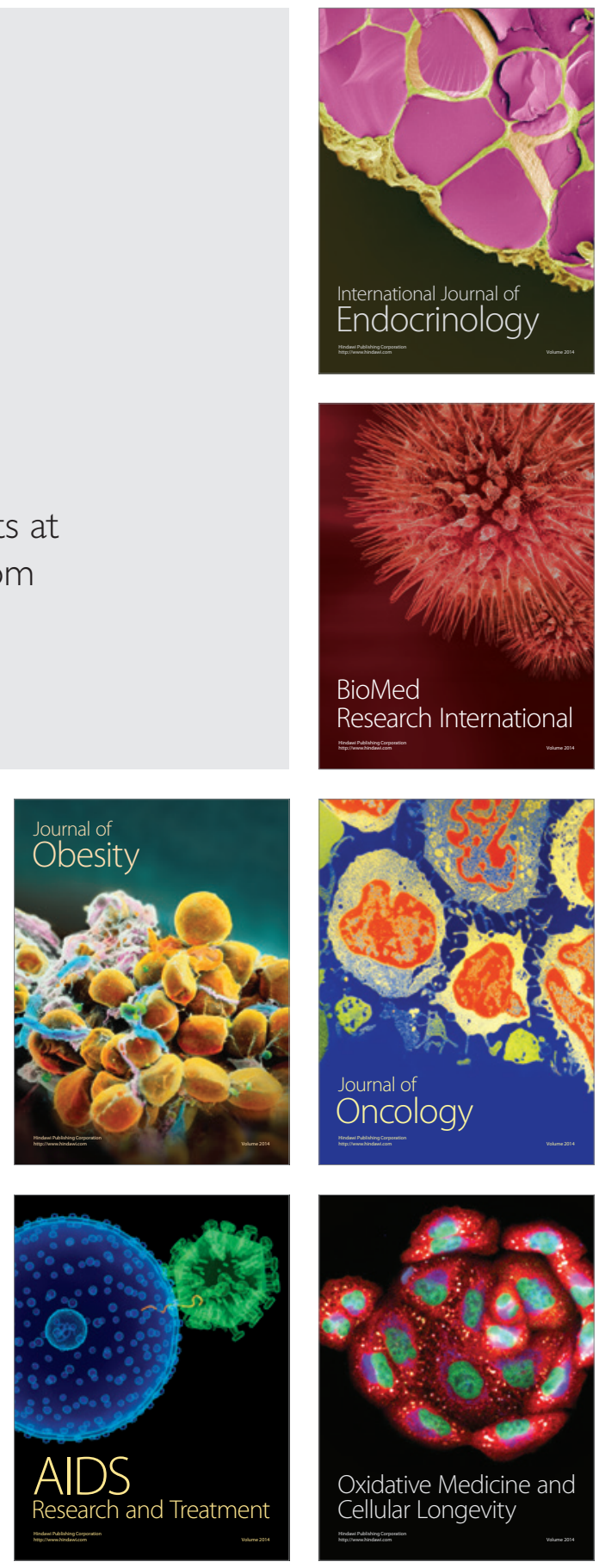